\title{
Adaptive Response Time for Integrated Autodriller Controller
}

\author{
Khim Chhantyal Kjartan Thor Birgisson Akash Padir* \\ National Oilwell Varco \\ khim.chhantyal@nov.com kjartan.birgissonenov.com \\ *Correspondence: akash.padirenov.com
}

\begin{abstract}
The integrated autodriller controller (IADC) is a software product by National Oilwell Varco (NOV) developed to allow an operator to drill with a constant weight on bit (WOB). The control loop has a proportional-integral (PI) controller, and PI gains are dependent on drill string compliance and response time (RT). The compliance is calculated based on the drill string length, but RT is difficult to predict. The existing IADC uses an empirical regression model to estimate RT based on the string length, which is not optimal during a formation change. This work presents an adaptive response time (ART), a machine learning model to predict RT based on the rate of penetration (ROP). ART can adapt to formation changes and gives smoother regulation of block velocity and WOB. Simulation results show a better torque and speed regulation of drill string using ART.
\end{abstract}

Keywords: oil \& gas drilling, integrated autodriller controller, adaptive response time, machine learning

Abbreviations: ART - Adaptive Response Time, CM Comprehensive Model, IADC - Integrated Autodriller Controller, NOVOS - National Oilwell Varco Operating System, PI - Proportional-Integral, ROP - Rate of Penetration, RPM - Revolutions per Minute, RT - Response Time, TD - Top Drive WOB - Weight on Bit

\section{Introduction}

The ability to maintain constant weight on bit (WOB), torque, and revolutions per minute (RPM) is essential to achieve optimal drilling efficiency for conventional drilling rigs. Control over these parameters maximizes bit life and increases ROP, thus resulting in reduced drilling completion time. Autodrillers are the commonly used tools in the drilling industry for WOB control by applying regulated force on the drill bit by means of a proportionalintegral (PI) controller. An industry-wide problem with autodrillers is maintaining regulation when drilling from one formation to another of different strength (Pastusek et al., 2016; Badgwell et al., 2018; Cayeux, 2018). This causes the error within the control loop to suddenly increase or decrease, resulting in controller instability. (Pastusek et al., 2016) pointed out the coupling between incorrect autodriller regulation and the drill string stick-slip. Operator intervention is required to stabilize the regulation, either by changing the setpoint or gain of the con- troller (Pastusek et al., 2016; Badgwell et al., 2018; Adam, 2018). Therefore making the success of the process dependent on the operator's skills.

In recent years, there have been several studies done to tackle this problem as the industry is shifting with an increased focus on autonomous drilling and limiting the need for human intervention. Adam (2018) collected autodriller parameters and corresponding ROP from successful drilling operations and created a road map intended to assist operators to select optimal autodriller parameters. Badgwell et al. (2018) proposed to use the ROP setpoint to auto-adjust the WOB gain to respond to autodriller instabilities. In addition, Badgwell et al. (2018) challenged autodriller vendors to address the WOB controller issues. We accepted this challenge.

Well dynamics are complex and well characteristics differ drastically between geological areas. Furthermore, lack of downhole sensors forces controllers to be regulated indirectly from top side equipment. Thus, developing general solutions with principle mechanistic models can be complicated. We therefore decided to use a data-driven approach like (Adam, 2018) in combination with the existing mechanistic model. Data-driven methods require data, which in the drilling industry is often proprietary. Obtaining a diverse dataset to ensure correct response from a machine learning algorithm in every scenario is also not feasible. For this reason, we used the approach of generating data by means of simulation.

\section{Methods}

\subsection{Simulation Environment Set-up}

The simulation environment consists of NOV's simulated integrated autodriller controller (IADC) and NOV's comprehensive model (CM). The IADC is an autodriller designed for regulating WOB, ROP, differential pressure, and torque limit. For this simulation, only the WOB mode (softWOB ${ }^{\mathrm{TM}}$ ) of the IADC is used. The CM is a simulation tool for drilling operations that contains fluid dynamics, string dynamics, formation characteristics, top side equipment, etc. to simulate well bore behavior. The IADC receives the WOB, ROP, top drive torque, and block position from the CM. Depending on setpoints and limits, the IADC adjusts the block velocity and feeds to the CM. Figure 1 shows a simplified block diagram of the simulation environment. 


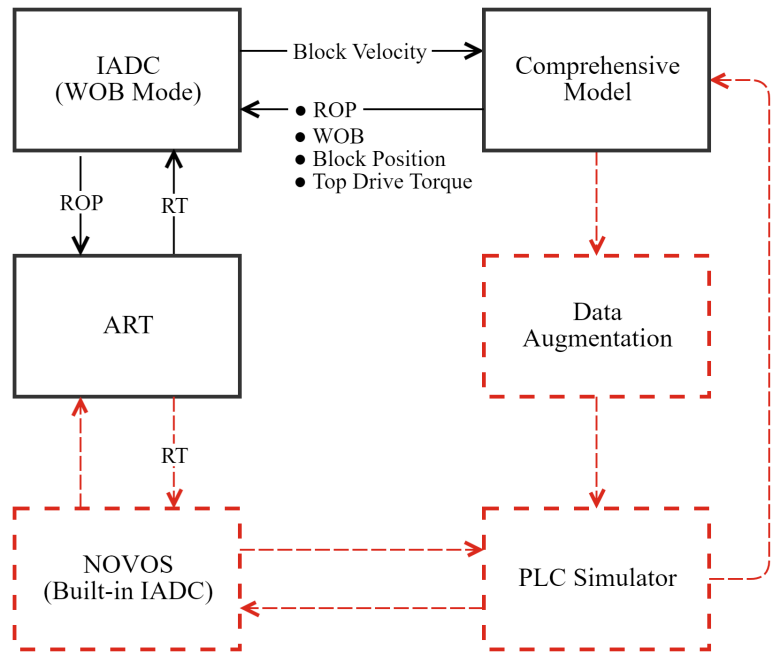

Figure 1. A simplified block diagram of the simulation environment. Current environment is displayed in black color, the NOVOS-based environment is displayed in red color (dotted lines).

Using the simulation environment, we created a dataset by performing drilling simulations through different formations in combination with various gains and depths. The dataset is used to train a machine learning based, Adaptive Response Time (ART) model. ART receives ROP as the only input from the IADC and predicts the response time (RT) for the controller. The IADC is currently executed in a PLC environment. The proposed ART is a polynomial model that can be implemented with minimal changes in existing installations and is ready for field testing.

ART is likely to improve the current IADC in a wide range of drilling scenarios. However, the problem is multidimensional and non-linear; therefore, the single input polynomial model is limited. To further improve ART, the feature space needs to be increased and a non-linear model implemented. This requires more drilling parameters and higher computational power. NOVOS is NOV's high level operating system, an automation platform built for full automatic control of the drilling process. Within NOVOS, ART has access to all possible drilling parameters and the computational power needed for training and executing non-linear models. The current simulation environment comprises of multiple mechanistic models. To increase the realism of the simulation model, we have added a simulator for all the equipment PLCs and a data augmentation layer for the signals coming from the CM to augment signal noise and variations. This environment will be used for simulations with the ART implemented in NOVOS. The NOVOS-based simulation environment is highlighted with red color (dotted lines) in Figure 1.

\subsection{Auto-Tune IADC}

IADC uses a PI controller to regulate WOB. Equation 1 shows the standard form of a PI controller.

$$
u(t)=K_{p} e(t)+\frac{K_{p}}{T_{i}} \int_{0}^{t} e\left(t^{\prime}\right) d t^{\prime}
$$

For IADC, the control variable $(u)$ is the block velocity and error $(e)$ is the difference between setpoint and calculated WOB. The proportional and integral gain parameters are function of drill string compliance and response time.

The drill string compliance is calculated based on the drill string length and mass. RT is a tunable time constant and can be set between 0.5 [s] and 20 [s]. It can be interpreted as a time the controller should use to adjust the block velocity to compensate for the error. As a general rule, RT should be tuned every 100-500 meters. Setting too short response time (closer to 0.5) implies an aggressive controller. This may cause the IADC to overreact on WOB changes and leads to increasingly large overshoots and oscillations. A high value of RT (closer to 20) implies slower response to WOB changes. It can tackle overshooting and reduce oscillations; however, it can take a long time to reach setpoint.

IADC currently offers functionality to estimate response time based on the drill string length. The function is a regression model based on empirical data from model analysis as well as real-world experiments. This approach does not account for formation type being drilled, resulting in unstable regulation caused by formation change. ART is designed to tackle this issue by detecting current formation using calculated ROP.

\section{Results}

In this section, simulation results of the IADC with traditional approach and with ART are presented. Table 1 shows the drilling parameters and ranges used in this study. As per traditional approach, RT (2.55 seconds) is selected based on the regression model for the given bit depth (2,050 meters).

Table 1. Drilling parameters and their ranges, used in the simulations.

\begin{tabular}{ll}
\hline Parameters & Range \\
\hline Drilling depth & $2,040-2,055[\mathrm{~m}]$ \\
ROP limit & $\pm 170[\mathrm{~m} / \mathrm{h}]$ \\
WOB limit & $\pm 25[$ tons $]$ \\
WOB setpoint & $20[$ tons $]$ \\
Drillability & $15-66[\mathrm{~m} / \mathrm{h}]$ \\
\hline
\end{tabular}

To analyse the simulation results, the following parameters are plotted against time in the subsequent subsections.

- WOB: operator setpoint vs. measured weight on bit

- MeasuredDepth: drilling progress against time

- Drillability: parameter describing relation between rock toughness and the bit's ability to produce hole. 

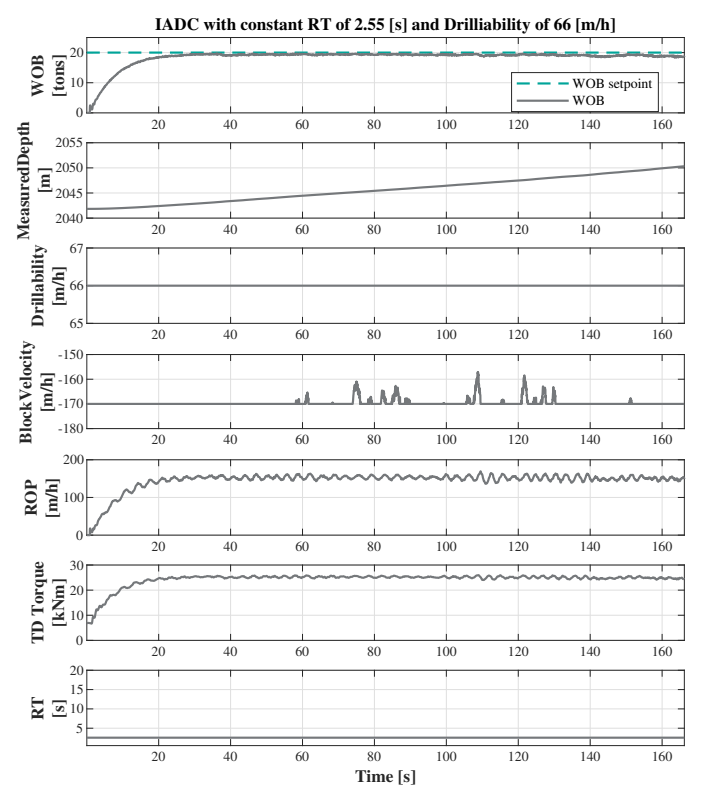

Figure 2. The performance of the IADC with constant RT = $2.55[\mathrm{~s}]$ and constant drillability $=66[\mathrm{~m} / \mathrm{h}]$.

A higher number results in faster drilling (higher ROP) for a given weight/speed on bit.

- BlockVelocity: block velocity due to controller action (positive/negative represents hoisting/lowering)

- ROP: calculated rate of penetration

- TDTorque: measured top drive torque

- RT: response time used by the controller

\subsection{Case 1: IADC performance in homoge- neous formation}

In this case, a homogeneous formation is chosen with drillability of $66[\mathrm{~m} / \mathrm{h}]$ (indicating soft formation) throughout the drilling simulation. The value of drillability is chosen such that it suits the traditionally calculated RT (= $2.55[\mathrm{~s}])$. Figure 2 shows that the WOB smoothly follows setpoint. In this ideal drilling set-up, acceptable torque and speed regulation are achieved.

\subsection{Case 2: IADC performance against chang- ing formations}

For this simulation, the drilling set-up is maintained as in Case 1 with the exception of formation. Here, formation is simulated to change from soft $(66[\mathrm{~m} / \mathrm{h}])$ to harder formation $(50[\mathrm{~m} / \mathrm{h}])$. It is noticed that the constant $\mathrm{RT}=2.55$ [s] does not suit the harder formation. As the RT is not suitable, it results in block velocity oscillations, causing oscillations in WOB, ROP, and top drive torque as shown in Figure 3. The results indicates that the controller needs to be tuned in an event of formation change.

By adjusting controller parameters (i.e., WOB setpoint or RT), the operator can overcome oscillations caused by
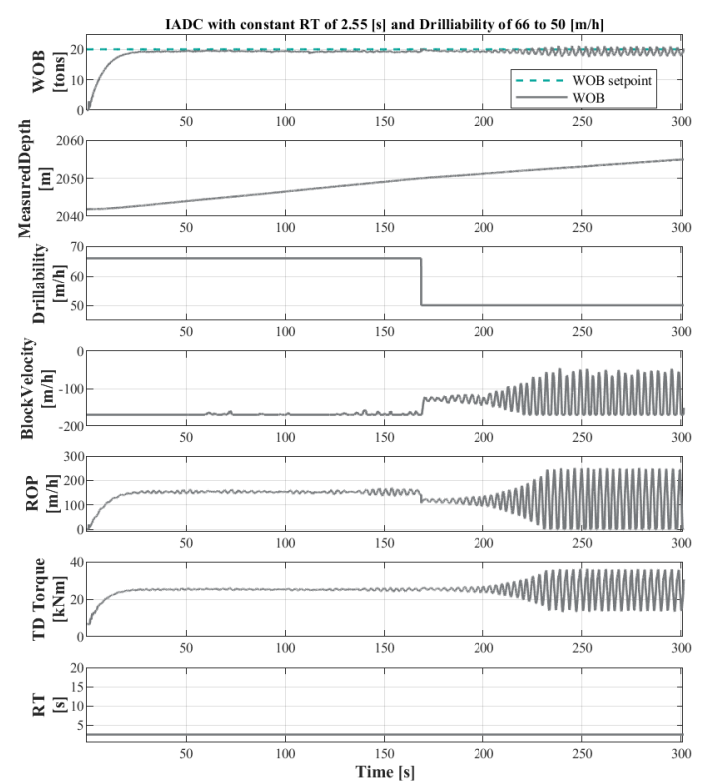

Figure 3. The performance of the IADC with constant RT = $2.55[\mathrm{~s}]$ and changing drillability from 66 to $50[\mathrm{~m} / \mathrm{h}]$.

formation change. In Figure 4, WOB setpoint is reduced from 20 [tons] to 10 [tons] and to 5 [tons] by the operator after noticing the oscillations. The oscillations reduce with changes in WOB setpoint. As the ROP is directly dependent on WOB, this results in reduced ROP. Hence, a proper way to reduce or eliminate oscillations and maintaining the ROP is by tuning RT for changing formation.

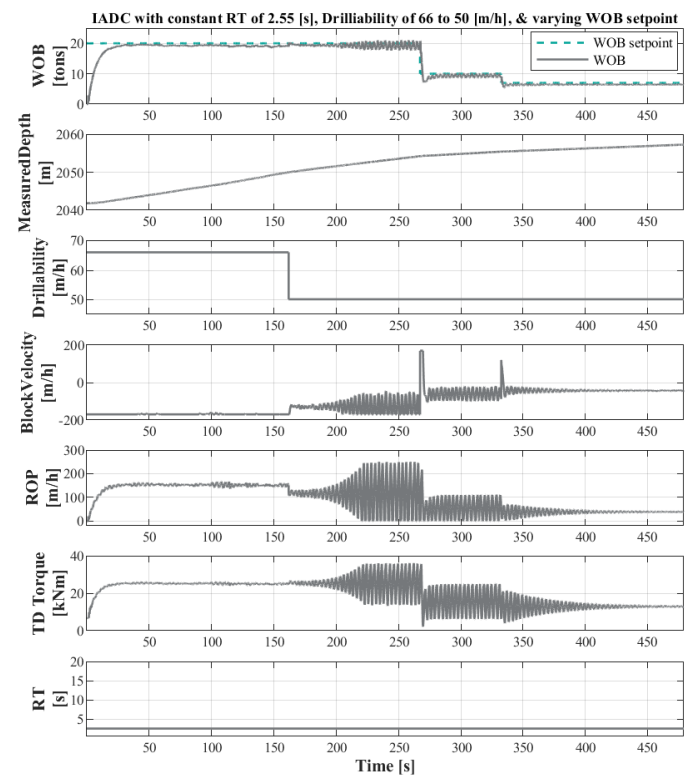

Figure 4. The performance of the IADC with constant RT = $2.55[\mathrm{~s}]$ and changing drillability from 66 to $50[\mathrm{~m} / \mathrm{h}]$. WOB setpoint is reduced to overcome the oscillations, which results in reduced ROP. 

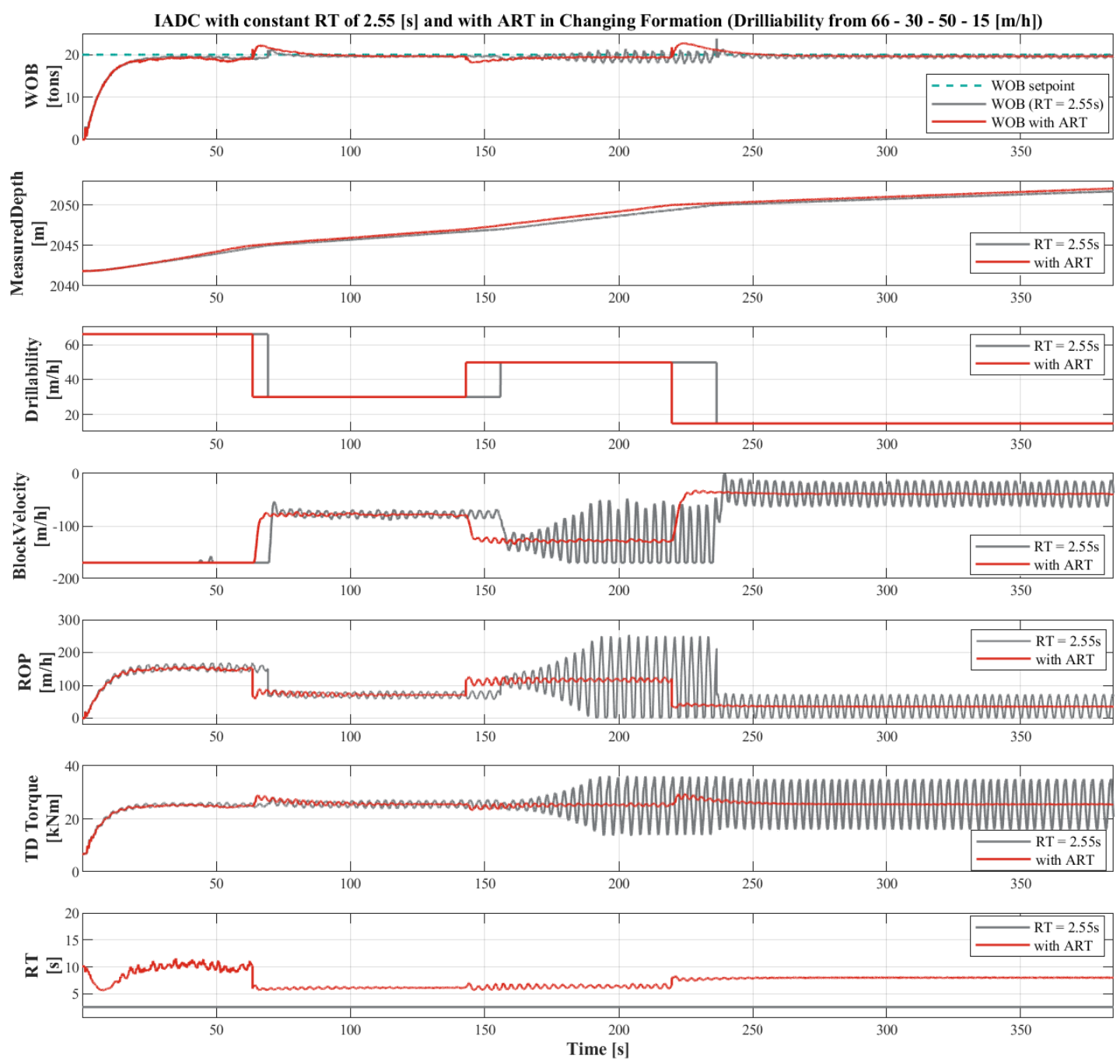

Figure 5. A performance comparison of the IADC with constant RT and with ART. IADC with ART can adapt to formation changes and provides smoother WOB, block velocity, and top drive torque. Stable top drive torque indicates elimination of autodriller induced stick-slip.

\subsection{Case 3: IADC with ART}

In Figure 5, drillability is randomly varied from 66 to 15 $[\mathrm{m} / \mathrm{h}]$ with increasing depth. ART can adapt to any formation change and selects a suitable RT based on ROP. The IADC with ART provides better regulation of WOB and stable block velocity. Improved regulation also causes top drive torque to stabilize, which is an indication of reduced stick and slip of the drill string. This ultimately results in increased drilling efficiency (increased ROP).

\section{Conclusion}

In the event of formation change, the existing regulator undergoes oscillations. These oscillations can be overcome by changing controller parameters (i.e., setpoint and gains). The current practice of manually changing the setpoints to tackle the oscillations, can lead to reduced drilling efficiency. Therefore, the changing formation should be addressed by tuning the response time. The presented machine learning algorithm based ART is capable of detecting the change in formation (by utilizing calculated ROP) and is able to estimate a suitable RT. During simulations, ART is found to stabilize the process to great extent, resulting in improved drilling efficiency.

Currently, ART is trained with the limited drilling range (1,000 - 3,000 meters depth). The presented simulations are performed within the trained range, but with different simulation set-up. ART further needs to be trained for the entire possible drilling range (up to 10,000 meters depth). In addition, ART can further be improved by adding features to the input feature space of the machine learning algorithm and with implementation of non-linear models in a high-level programming environment. 


\section{Acknowledgment}

The authors would like to thank NOV for the permission to publish this paper. As well as NOV's Robotic Control team, NOV's NOVOS team, \& NOV's R\&D for the collaboration.

Special thanks to Matthew Jackson, Francisco Ocegueda-Hernandez, Ivan Rajic, Njål Sama, Charles Holst Sandnes, and Morten Ørevik for their support.

\section{References}

D Adam. Reducing stick-slip by avoiding auto-driller control dysfunction. In IADC/SPE Drilling Conference and Exhibition. Society of Petroleum Engineers, 2018. doi:10.2118/189653-MS.

T Badgwell, P Pastusek, and K Kumaran. Auto-driller automatic tuning. In SPE Annual Technical Conference and Exhibition. Society of Petroleum Engineers, 2018. doi:10.2118/191417MS.

E Cayeux. On the importance of boundary conditions for real-time transient drill-string mechanical estimations. In IADC/SPE Drilling Conference and Exhibition. Society of Petroleum Engineers, 2018. doi:10.2118/189642-MS.

P Pastusek, M Owens, D Barrette, V Wilkins, A Bolzan, J Ryan, K Akyabi, M Reichle, and D Pais. Drill rig control systems: Debugging, tuning, and long term needs. In SPE Annual Technical Conference and Exhibition. Society of Petroleum Engineers, 2016. doi:10.2118/181415-MS. 